\title{
Research on the Stability of Power Supply in Electronic Communication
}

\author{
Yuan Ke \\ Nanchang Institute of Science \& Technology
}

Keywords: Electronic communication; Power supply; Stability

\begin{abstract}
Along with the progress of the social economic development and the continues improvement of information technology, China's communications industry has also rapidly developed. In the current application of communication power, with the superior characteristics, such as high power conversion efficiency, wide voltage range and light weight, electronic communication has technically reformed traditional linear power supply. The power supply of electronic communication is the core of the system and it plays key role in the operation of the whole system. In this paper, the stability of current electronic communication power supply is mainly researched with hope to play a certain role in promoting the development of the field.
\end{abstract}

\section{Introduction}

In the new historical stage of development, China's electronic information technology has been greatly developed and the process for electronic communication power supply stability have important research significance, but the degree of attention in this respect is not enough, people only pay attention to the electronic communication equipment. Therefore, the stability of power supply must get people's attention, which can effectively eliminate the hidden troubles caused by power supply.

To keep the safe and stable operation of power system communication network, one of the important conditions is the communication power supply. One of the important means to improve the stability of power communication system is to reduce the incidence of accidents of communication power supply. There are many problems caused by old power supply systems which are used in the current electric power communication system. Aiming at this phenomenon, this paper introduces the basic situation and existing problems of the development of electronic communication power supply, and mainly puts forward the ideas and suggestions to better maintain and operate the communication power supply.

\section{Basic Overview of Electronic Communication Power Supply}

Communication power supply system is the core of the communication system, and the stable and reliable communication power supply system is the key to guarantee the safety and the reliable operation of communication system. Once the communication power supply system failures which will cause the interruption of the power supply of communication equipment, communication equipment will not run, which then causes the interruption of the communication circuit and the standstill of the communication system, resulting in great economic and social benefit loss. Therefore, communication power supply system occupies very important position in communication system.

In the electronic communication system, communication power supply is the most important part, which can be said that is the heart of the electronic communication system. Only a stable power supply for the system can make the system operate safely and normally. If the power supply of 
communication system is instability or due the power supply fails, electronic communications equipment cannot run, which will cause great economic loss. However, from the point of view of the development of the communication power supply, the power supply in electronic communication does not account a large proportion in the industry. The system of electronic communication power supply is mainly composed by AC and DC power supply, rectifier cabinet and monitoring module.

\section{Analysis of Power Stability in Current Electronic Communication}

In recent years, with the progress of technology, especially updated power, the constant use of new electromagnetic materials, the continuous improvement of power conversion technology, continuous progress of control method, and the constant fusion of related disciplines technologies, communication system has made considerable progress in the reliability and stability of system, electromagnetic compatibility, elimination of the harmonic of the current on line side, and improving the utilization rate of electric energy, reducing loss, improving the system dynamic performance, etc.

Although the electronic communication technology of our country is in development, in the power supply stability, there are still some shortcomings. The most common problem is that its reliability and stability are not enough and the lightning protection measures still need to be further improved. In addition, the power device is easy to damage. The technical content also has its own shortcomings and it is in lack of competitive power. The current self turn off devices is mainly rely on imports and testing means are lacking. From the point of view of the current development situation, the design of communication circuit still relies on the designer's personal experience and skills. Therefore, it is not systematic and the understanding of the electromagnetic compatibility is not enough.

\section{Research on the Stability of Power Supply in Electronic Communication}

Establish Monitoring System and Maintain the Stability of Power Supply. A single set of power communication equipment is relatively simple, which is generally equipped with the machine monitoring device and real-time monitoring to communication unit, DC unit, battery and other working states will be carried out, including the system voltage, the working condition of the system, including the system voltage, the display of the working condition of the system, the outputting current of the load current and the rectifier module, the current and $\mathrm{AH}$ of charging and discharging of battery, the setting values of each operation parameter of the system, the battery temperature and the ambient temperature. According to different kinds of setting values, send all kinds of alarm information. For emergency failure, there is a call-back function, which can notify the operator immediately, the composition of which is as shown in Fig. 1: 


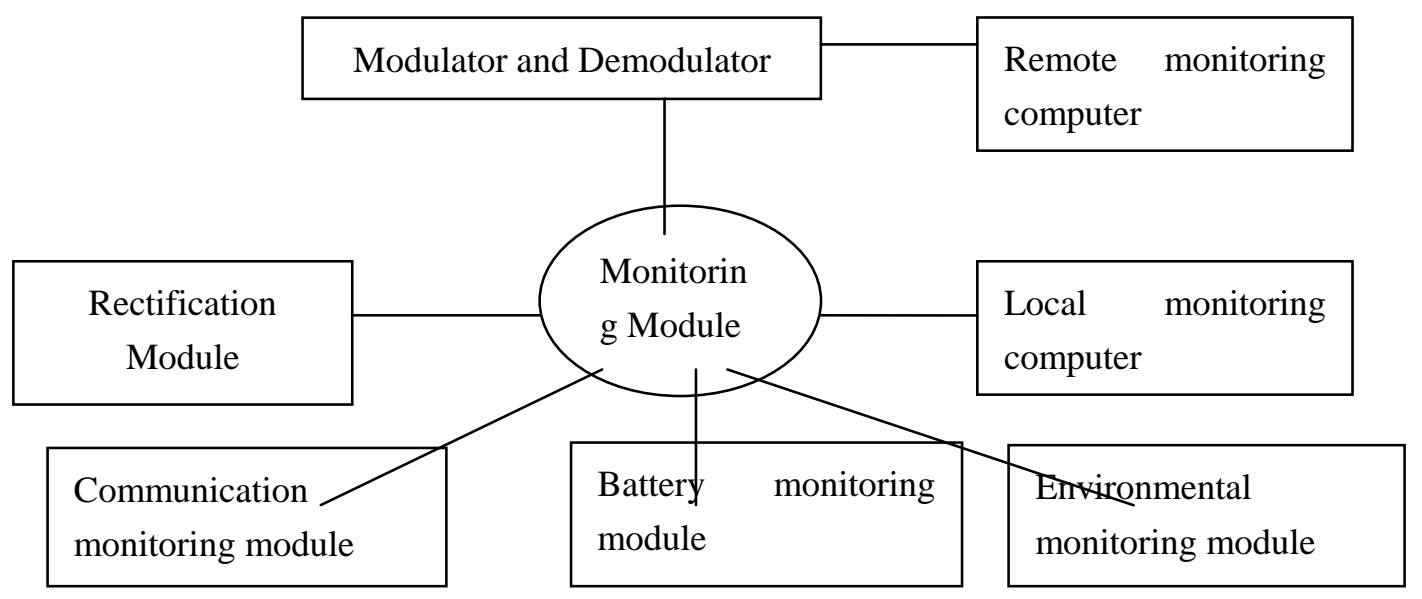

Figure. 1 Supervisory control system

Maintain the Stability of the Power Supply through the Battery. Communication power supply shall be carried on effective maintenance, for example to check whether there is aging or damaged outside the power supply or whether there is active material shedding. During using, the regular discharge test shall be done to the power supply. The discharge amount shall be $30 \%$ to $40 \%$ of the total capacity of the power supply. The resistance of power supply shall be tested regularly. As the time passing by, the service life of the power supply will bring greater resistance to the power supply. Knowing such situation will be very helpful to the stability of communication power supply.

Communication Personnel Quality Training. The personal qualities of the management personnel of communication power supply shall be further strengthened. There will be a variety of technology for the production of electronic communications power supply which will apply. For example: the power electronic components and semiconductor and other related devices. Therefore, in addition to do well in the maintenance and management of the electronic communication power supply, the personnel with the relevant knowledge and professional abilities shall be managed, which requires to improve the qualities of the management personnel of this field. So, only to guarantee the security of communication power supply can the stability of the power supply be improved effectively and the people's communication works run smoothly.

\section{Conclusion}

With the rapid development of electronic communication technology currently, the stability of the power supply shall be effectively improved. Power electronic technology is an important basis for scientific and technological support, which has good protection function to the power supply stability of electronic communication. Now the communication technology develops in the diversification forms and communication products appear endlessly. However, these aspects of development do not have a role in reducing the power supply. The stability of the power supply cannot improve, the equipment will not function properly. Therefore, to solve the problem of the stability of power supply electronic communication is the key.

\section{References}

[1] Nagai M. Power supply for switching operation, electronic apparatus including the same, and method of controlling the same: US, US7315159 [P]. 2008. 
[2] Sun M J, Xu Z Q, Tu Y N, et al. Research on the stability of CWS based on the multiple light scattering principle [J]. Meitan Xuebao/journal of the China Coal Society, 2015, 40(3):659-664.

[3] Huang H, Zeng T, Zhang T, et al. Research on the stability of large aperture mirror mounts using cross-flexure pivots in ICF lasers [J]. Chinese Optics Letters, 2007, 5(s1):S60-S63.

[4] Yang H, Li L, Wang D. Research on the Stability of Open Financial System [J]. Entropy, 2015, 17(4):1734-1754.

[5] Shahbazi M, Wang H. Progress in research on the stability of organometal perovskite solar cells [J]. Solar Energy, 2016, 123:74-87.

[6] Jong W D. Experimental research on the stability of the armour and secondary layer in a single layered tetrapod breakwater [J]. Civil Engineering \& Geosciences, 2004.

[7] Wu C, Wang W, Shi H, et al. Research on the Stability of Vehicle Suspension Excited by the Road Surface Profile[C]// Computer Modeling and Simulation, 2010. ICCMS '10. Second International Conference on. IEEE, 2010:164-168.

[8] L'Hermite F. Method and apparatus for reducing audible acoustical noise in a power supply transformer by shaping the waveform of a primary side inductor current: US, US 6477066 B2 [P]. 2002.

[9] Fabian M R, Sonenberg N, Filipowicz W. Regulation of mRNA translation and stability by microRNAs. [Review] [189 refs][J]. Annual Review of Biochemistry, 2010, 79(1):351-379.

[10]Gale D. College Admissions and the Stability of Marriage [J]. American Mathematical Monthly, 2013, 69(69):9-15.

[11]Connors K A. The Stability of Cyclodextrin Complexes in Solution [J]. Chemical Reviews, 1997, 97(5):1325-1358.

[12]Botwinick J. Personality development: stability and change. [J]. Annual Review of Psychology, 2005, 56(1):453-84. 